\title{
25001
}

UCRL-ID-127772

\section{Real-time Software Use in Nuclear Materials Handling Criticality Safety Control}

\author{
S. Huang \\ D. Lappa \\ T. Chiao \\ C. Parrish \\ R. Carlson \\ J. Lewis \\ D. Shikany \\ H. Woo
}

June 1, 1997

This is an informal report intended primarily for internal or limited external diatribution. The opinions and conclusions stated are those of the author and may or may not be those of the Laboratory.

Work performed under the auppices of the U.S. Department of Energy by the Lawrence Livermore National Laboratory under Contract W-7405-Eng-s8. 


\section{DISCLAIMER}

This document was prepared as an account of work sponsored by an agency of the United States Government. Neither the United States Government nor the University of California nor any of their employees, makes any warranty, express or implied, or assumes any legal liability or responsibility for the accuracy, completeness, or usefulness of any information, apparatus, product, or process disclosed, or represents that its use would not infringe privately owned rights. Reference herein to any specific commercial product, process, or service by trade name, trademark, manufacturer, or otherwise, does not necessarily constitute or imply its endorsement, recommendation, or favoring by the United States Government or the University of California. The views and opinions of authors expressed herein do not necessarily state or reflect those of the United States Government or the University of California, and shall not be used for advertising or product endorsement purposes.

This report has been reproduced directly from the best available copy.

Available to DOE and DOE contractors from the Office of Scientific and Technical Information

P.O. Box 62, Oak Ridge, TN 37831

Prices available from (615) 576-8401, FTS 626-8401

Available to the public from the

National Technical Information Service

U.S. Department of Commerce

5285 Port Royal Rd.,

Springfield, VA 22161 


\title{
Real-time Software Use in Nuclear Materials Handling Criticality Safety Control
}

\author{
S.Huang, D. Lappa, T. Chiao, \\ C. Parrish, R. Carlson, J. Lewis, D. Shikany, and H. Woo \\ Lawrence Livermore National Laboratory \\ P. O. Box 808 \\ Livermore, CA 94551
}

\subsection{Introduction}

All LLNL operations involving significant quantities of fissionable material conform to the double contingency principle for criticality safety. The typical order of preference for criticality safety control is: (1) passive engineered controls, such as favorable geometry in equipment and spacing; (2) active engineered controls; and, (3) administrative controls. In many instances, administrative controls are employed due to practicality and cost considerations.

Protection against an inadvertent criticality incident is provided through either: (1) the control of two independent process parameters, as the preferred approach; or, (2) through a system of multiple controls on a single parameter. Administrative control generally relies on written procedures and human operator compliance. For example, the two-person rule on the control of fissionable material mass limits has been used in various facilities as a means of meeting the double contingency principle.

This paper addresses the use of real-time software to assist handlers of fissionable nuclear material. We focus specifically on the issue of workstation mass limits, and the need for handlers to be aware of, and check against, those mass limits during material transfers. Here "mass limits" generally refer to criticality safety mass limits; however, in some instances, workstation mass limits for some materials may be governed by considerations other than criticality, e.g., fire or release consequence limitation.

As a case study, we provide a simplified reliability comparison of the use of a manual two handler system with a software-assisted two handler system. We identify the interface points between software and handlers that are relevant to criticality safety.

\subsection{Description of Software for Mass Limit Control}

At LLNL, software has been used a part of a system for tracking nuclear material for safeguards and security. The software tracks the movement of items and provides the current inventory at each workstation. For several years, this software has been used to assist handlers in observing the workstation mass limits.

The software requires independent entries by two handlers for all transfers. It also requires both handlers to independently verify the fissile material inventory before and after each transaction. Therefore, two handlers validate the mass limit for a transfer. This provides two nominally 
independent controls on the same parameter (mass). The loss of one control on mass would not allow the mass limits to be exceeded; therefore, double contingency is met.

LLNL's software was originally designed for use in material control and accountability (MC\&A). In order to effectively use the software for criticality safety control, additional improvements are being planned. Specifically, the improved software should consist of three parts: 1) an inventory module to track mass inventory; 2) a mass limit module to provide mass limits for each workstation; and, 3) an interface module to provide the interface between the inventory module, the mass limit module, and the handler.

The inventory module should update the material inventory database as material transfers occur. It should also provide, on request, the current inventories for a given workstation.

The mass limit module should address all current appropriate mass limits, including limits for the various operating conditions, dispersible components, box loss, and isotopic compositions for a given location. The mass limits should be defined by the approved operational safety procedures (OSPs) and entered/maintained independently from material handlers. Any change to the software's mass limits database should be made only after a formal change to the corresponding OSP. Handlers should determine which operating condition is in effect for an operation, and they must be able to verify the mass limit provided by the software against the corresponding OSP.

The interface module should be the only link between the inventory module and the mass limit module. The interface module should obtain the current workstation inventory from the inventory module, add the mass of the proposed transaction (provided by the handlers), and compare against the mass limit provided by the mass limit module. If the proposed transaction violates the mass limit, the interface module should provide a real-time warning to the handlers and the proposed transaction should be administratively denied by the software.

The interface module should provide for "two person" control, and should log all transactions. Such control should require the second handler to $\log$ in his or her ID and password to authorize the transaction, and also some transaction attribute information. This may include: the destination workstation number, the serial number of the transferred item, an explicit confirmation that the proposed inventory is within the mass limit, etc. The log should include all transaction requests, including especially those rejected by the software.

\subsection{Comparative Evaluation}

\subsection{Method of Evaluation}

The comparison of manual versus software-assisted mass control systems is significantly complicated by the human elements in each system. The wide variability in human performance, the complexity of analyzing human failure, and the dearth of significant relevant databases can make the quantitative reliability analysis of such systems difficult, if not impossible.

However, there are objective methods for evaluating the reliability of systems involving human action, and, more importantly, the uncertainty in performance data is more problematic to absolute assessments of reliability than to relative comparisons such as this one. There is a significant body of literature dealing with many aspects of reliability analysis, human performance, etc., and we do not attempt to summarize it here. That literature makes clear there are continuing needs to gather validated databases on the reliability of complex systems involving human operators. 
In this comparative evaluation, we do not address the reliability of manual and software-assisted mass control systems in detail. Instead, we describe such systems generally, and focus on those details most relevant to differences in reliability of the two systems.

\subsection{Manual Mass Control System}

\subsubsection{General Description}

As the name implies, this class of systems is highly or exclusively dependent on human action to control mass. A typical example of such a system is one employing log sheets or "blue cards" for each workstation, and on which material handlers and/or their supervisors make handwritten entries for such quantities as: the present inventory of a specific material type in that workstation; the amount of that material type being transferred into or out of a workstation; the resulting inventory; and administrative notations, such as checkbox markings and signatures, to indicate that the resulting inventory is within mass limits for the workstation and that there has been multiperson control of the operation.

One of the most significant aspects of a manual control system is that all arithmetic involved with calculating transfer masses and resulting masses is carried out by humans, presumably with the aid of basic computing aids like hand calculators.

The specific process for mass control calculations can vary, depending on the degree of redundancy that system designers seek to establish. For example, a system could require that two handlers independently, using different techniques and without interaction, calculate the masses involved with a proposed operation and evaluate its appropriateness, communicating their findings with each other only when both have completed their assessment. By contrast, a system could have a primary handler carry out the assessment, and a supervisor "check" the completed assessment for validity; or, two handlers could together carry out the assessment, with the second handler monitoring, at each step, the accuracy of the primary handler's calculations; or, etc.

\subsubsection{Reliability Considerations}

For this system, the large dependence on human action in conformance with administrative controls is a potential weakness. Depending on the specific design characteristics, irrespective of personnel quality and training, such manual systems will have greater or less reliability by virtue of the degree of true redundancy derived from human action.

The degree to which the use of multiple personnel enhances reliability is highly dependent on the correlation of failure of those personnel. Among the systems described in Section 3.2.1, some human actions may be more independent of other redundant human actions, if opportunities for correlation are reduced or eliminated. Thus, having two handlers, employing different techniques, redundantly assess the viability of an operation is likely superior to having them work as a team.

One of the intrinsic weaknesses of a manual two handler mass control system is that the system is prone to common cause failures, such as: a supervisor's influence over conduct of operations; handler susceptibility to circumventing procedures when under schedule pressures; and, the potential to confuse details in multiple workstation environments.

\subsection{Software-assisted Mass Control Systems}

\subsubsection{General Description}


In these systems, designers have attempted to improve reliability, reduce costs, increase productivity, etc. by employing computer technology to complete one or more functions carried out by humans in a manual mass control system. For example, the manual system described in Section 3.1 is actually mildly computer-assisted by virtue of the hand calculator -- a computer -being used to carry out simple arithmetic operations which otherwise would be done using pencil and paper. [We nevertheless regard the system in 3.1 as "manual" because, over the past few decades, the use of hand calculators has been deemed, appropriately or not, to be superior to longhand arithmetic for all but the most trivial calculations. Most people would not readily identify their pocket calculator, wristwatch, etc. as an electronic computer because those objects have entered the realm of "tools", i.e., low-tech and highly reliable; while "computers" are now deemed to be overly complicated and unreliable objects like PCs and programmable VCRs!]

For our discussion, "software-assisted" refers to more than the employment of hand calculators and other simple devices. Specifically, it means the use of one or more CPUs, running compilable software, employing non-volatile databases, and accessed through a human-machine interface such as a keyboard, mouse, and monitor.

There are a variety of ways in which real-time software can be employed to assist with mass control. The most obvious and, probably, the most common way is to employ software to carry out those functions to which computers are best suited. Computers tend to be far more reliable than humans at: storing and retrieving large quantities of numeric data; rapidly carrying out numerous, complex arithmetic calculations with great precision, including comparative operations; monitoring a data stream continuously for interesting characteristics; and so on.

Consequently, one of the most prevalent uses of software in fissile material operations today is in safeguards and security. Such MC\&A systems are employed to keep track of the type and amount of material in various locations in a safeguarded facility. If those locations are defined to be the same level of workstation at which criticality or other mass limits are imposed, then the potential utility of such a software-based MC\&A system to mass control is self-evident. If handlers are being administratively required to employ a software-based MC\&A system to log all material transactions, then additional software and data can extend that MC\&A system to one that also monitors workstations for the potential of exceeding mass limits.

\subsubsection{Reliability Considerations}

The reliability of a software-assisted administrative control largely depends on the design details of the system and its implementation within the overall criticality safety control infrastructure at a facility. Perhaps the single most important factor affecting the reliability of a software-assisted mass control system is whether the software-based functionality is added to supplement or replace human-based functionality. Borrowing from the systems described under section 3.1, consider two handlers who independently assess the viability of a transaction before comparing their results. If the system is modified so that, after this process, the handlers then are required to carry out the transaction via an MC\&A system with a mass limit checking function, then the system reliability is likely improved by the additional computerized operation. However, if the use of computerized mass limit checking means that procedurally, or in practice, handlers no longer carry out their redundant and independent assessment of transaction viability, then system reliability may be degraded.

One of the clear advantages of a software-assisted mass control system is the ability of the software to provide a real-time warning when a proposed mass transfer would violate the mass limit for a workstation. The benefit of such a real-time warning from a reliability perspective is that it is passively initiated, i.e., handlers are not required to check some condition in order to be alerted to 
the danger. The warning is automatic, and derives from other actions which the handlers take as part of routine operations.

\subsection{Comparison of Manual and Software-assisted Reliability}

Detailed, direct comparison of two such systems is possible only with fully developed systems descriptions. Here, we are dealing with more general aspects of manual and software-assisted mass control systems. Thus, our comparison is general in nature, though some specific points of comparison based on hypothetical details is offered.

\subsubsection{Double Contingency Principle}

A natural basis for weighing the advantages of computerizing criticality safety control procedures is the double contingency principle, as found in DOE Order 420.1:
"Process designs shall incorporate sufficient factors of safety to require at least two unlikely, independent, and concurrent changes in process conditions before a criticality accident is possible. Protection shall be provided by either (i) the control of two independent process parameters (which is the preferred approach, when practical, to prevent common-mode failure), or (ii) a system of multiple controls on a single process parameter. The number of controls required upon a single controlled process parameter shall be based upon control reliability and any features that mitigate the consequences of control failure."

A key requirement here is for "independent" changes. This basis alone argues for a robust criticality safety control employing diverse safety features. Systems that are highly dependent on multiple handlers and/or supervisors, all subject to common modes of human failure and, therefore, common cause failure under similar or identical operating conditions, are likely to have less defense-in-depth than systems which depend on a combination of human and "mechanical" safety features. (Here, "mechanical" means features that are based in hardware, including electronic computers, etc., with little or no active human participation in the safety function of that feature.)

\subsubsection{Controls Reliability}

The number of controls required for a single process parameter is dependent on "control reliability". To strengthen a control reliability, one needs to examine the key features contributing to the reliability of that control, and seek to strengthen each of those individual features. Thus, for a feature involving human action, factors like: education, training, and experience; performance monitoring and metrics; real-time performance feedback; environmental factors; morale; etc. are all important to reliability. Humans are much more prone to make arithmetic errors than, say, a welldeveloped software application platform. Where operations involve the retrieval and manipulation of numerical databases, like material masses in a critical mass control process, there is likely to be significant benefit from the employment of a computerized safety feature in parallel with a humanbased assessment of transaction viability.

Whether this argues for the use of two, redundant, software-assisted assessments as superior to one manual and one computer-assisted assessment is doubtful. As with human actions, computerbased actions are also prone to failure from common modes or causes, such as software quality assurance errors, data entry errors, hardware malfunction, human-machine interface failure, etc. Although this weakness can be reduced by proper system design and quality assurance, extensive measures of this kind can be very expensive. 


\subsubsection{Additional Considerations}

The use of a software-assisted mass control safety feature may provide additional improvements to overall system reliability than those discussed above. Given the ease with which transaction data can be captured, stored, and processed -- even in real-time -- by modern computer systems, there is enormous potential for software to be employed to monitor materials transactions, including: the timing, frequency, and type of transactions; material quantity and type distributions; handler calculation error; handler duty factors; etc. The list is long, indicating the potential utility of software-assisted safety features to improve overall system reliability and reduce the risk of inadvertent criticality.

\subsection{Case Study}

We here provide an analysis of two distinct systems for carrying out the same criticality control function; namely, the determination of mass limit violation for a proposed nuclear material workstation transaction. Such mass limits should be quite familiar to materials handling facility personnel. They are developed for workstations based on various considerations, including, most relevant here, the need to avoid the aggregation of a potential critical mass in a material workstation. With respect to criticality safety, such limits must account for issues of material type, moderator/reflector presence, etc.; and, consequently, because of changing conditions in material handling workstations, there may be multiple applicable mass limits for any given workstation. When material is transferred into a workstation, care must be taken that the resulting inventory does not exceed the appropriate mass limit for that workstation.

\subsubsection{Systems Studied}

The first system involves two handlers independently and manually assessing transaction viability by checking printed documentation of a workstation's inventory and mass limit. These might be posted on the workstation or in an OSP kept near the workstation. They each manually calculate the workstation inventory resulting from the intended transfer and compare that against the mass limit.

The second system also involves two handlers determining workstation inventory; one through the method for the first system, and the second through the use of software that tracks material inventories. In this system, the software determines the workstation mass limit from a database of such limits, and then automatically compares the proposed inventory against that mass limit. It then advises the handlers if a proposed transaction.would result in a mass limit violation.

\subsubsection{Reliability Analysis}

Figures 1 and 2 provide highly simplified fault trees describing the failure of each of the systems studied. Though simplified, the trees provide an example of some fundamental reliability aspects of the two systems for checking mass limits.

In the first system, the two handlers independently: 1) calculate the proposed inventory of the workstation; 2) determine the appropriate mass limit for the workstation; and, 3) compare the proposed inventory against the mass limit to determine the proposed material transfer's acceptability. The presumption in this tree is that if either handler errs, the other handler's correct assessment will lead to conflicting conclusions about acceptability and that this, in turn, will lead to a careful and correct assessment of unacceptability. Both handlers must err in order for there not to be a conflict of opinion and, consequently, the exceedance of the mass limit. Thus, the top event gate is controlled by an AND gate. 
This fundamental aspect of the failure of the two handler system is essential to arguments in favor of a software-assisted system. The degree to which true redundancy is achieved depends on the degree of independence of the branches to the AND gate. If handlers' errors are correlated in some way, that is, if there are common mode or common cause failures, then the overall probability of system failure will be accordingly increased. In the extreme, if the two handlers' failures are perfectly correlated, the presence of the second handler may provide no additional reliability.

Figure 2 provides the fault tree for the software-assisted system. Note that it too is governed by a top event AND gate. Thus, both the manual check and the software-assisted check of the mass limit must fail for the mass limit to be exceeded. Unlike the manual system, the highly simplified basic events (perhaps more appropriately modeled as undeveloped events) are quite different between the two branches. This is more than an artifact of terminology. It is clear that the procedures, equipment, data, etc. that would be employed in carrying out the functions associated with the right branch in Figure 2 are likely to be rather distinct from those of the left branch.

This does not mean that there are not mechanisms for correlated failure between the two branches. A good example of such would be the erroneous underassessment of the amount of fissile material contained in a part to be transferred. In such a case, both the manual assessment of the final inventory and the software-based assessment of that inventory would be low, leading to a potential mass limit violation. Nevertheless, the failures necessary between the two branches of the software-assisted system are more distinct and, therefore, less prone to common cause/mode failure than for the manual system.

Without specific description, in an appropriatley detailed tree, of the failure probablities associated with the basic events, it is not possible a priori to state which of these systems has greater reliability. For example, even with significant correlation between handler failures in the two handler system, if the error rates of the handlers in any of the steps involved is far lower than the error rates in a poorly designed and implemented software system, then the two handler system could well be more reliable than a software-assisted system.

\subsection{Conclusions}

Using software to track nuclear material for control and accountability is not a new concept. DOE and other government facilities have employed software for this purpose for many years. Moving beyond MC\&A, and using the real-time software for criticality safety control is a potential next step for material handling facilities. At LLNL, real-time software is being used in both MC\&A and criticality safety control. The decision to implement or evolve software used for criticality safety control must be taken with many considerations in mind, including, primarily, the reliability and safety of the resulting criticality safety control system. 


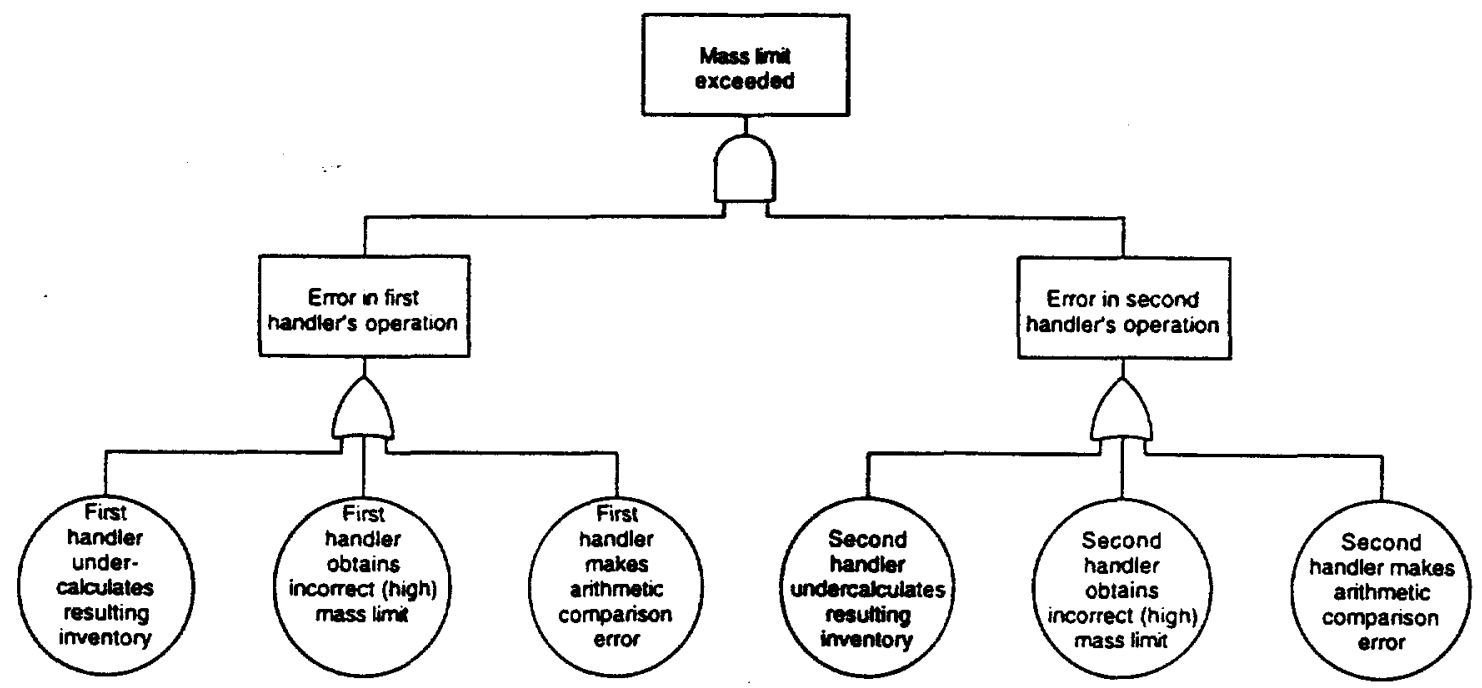

Figure 1. Manual System Fault Tree

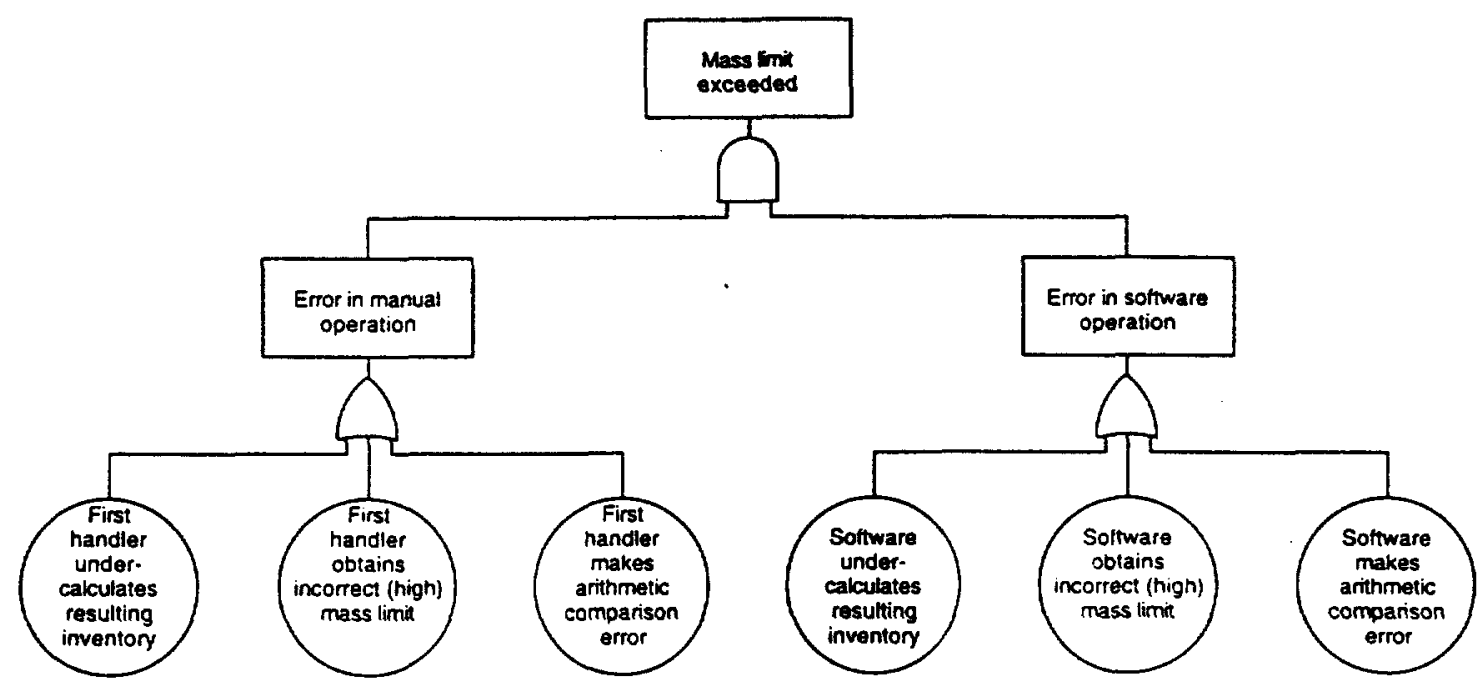

Figure 2. Software-assisted System Fault Tree 


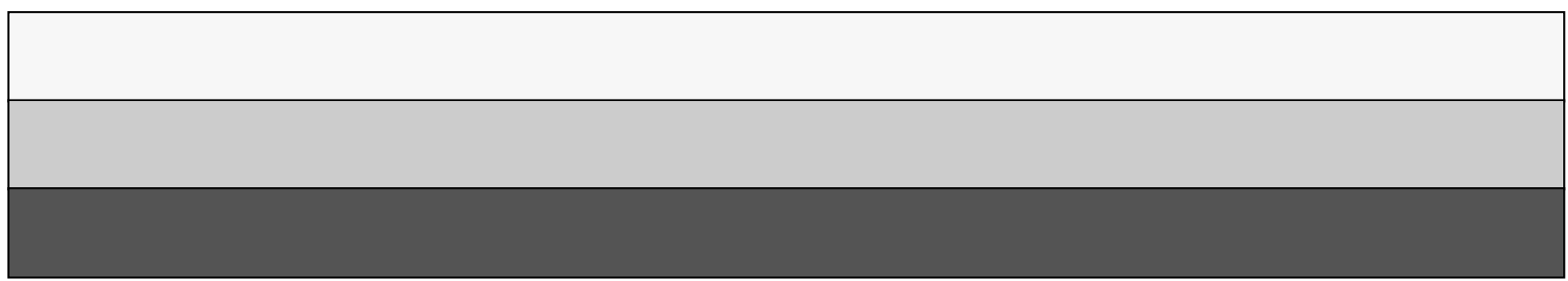

